\title{
A COMPLETE ORTHONORMAL SYSTEM OF HOMO- GENEOUS POLYNOMIALS ON MATRIX SPACES OF ORDER 2
}

\author{
JOSEPHINE MITCHELL ${ }^{1}$
}

1. Introduction. Let

$$
z=\left(\begin{array}{ll}
z_{1} & z_{2} \\
z_{3} & z_{4}
\end{array}\right)
$$

be a matrix of complex numbers, $z^{*}$ its conjugate transpose and $I$ the identity matrix. Let $D$ be the domain $E\left[z \mid I-z z^{*}>0\right]$. The set $B=E\left[z \mid z z^{*}=I\right]$ forms a proper part of the boundary of $D$. Hua $[3 ; 4]$ has shown by means of representation theory that there exists an orthonormal system (ONS) of homogeneous polynomials in $D$ with respect to the inner product $\int_{D} f \bar{g} d V_{D}$. In this paper we exhibit such a system $\left\{\phi_{j}\right\}$ explicitly on $D$ in a special case, only with respect to the inner product

$$
\left(\phi_{j}, \phi_{k}\right)=\int_{B} \phi_{j} \bar{\phi}_{k} d V=\delta_{j k}
$$

( $d V$ Euclidean volume element on $B$ ). The functions turn out to be certain hypergeometric functions multiplied by powers of $z_{j}$.

2. Calculation of the inner product.

Lemma. $A$ parametrization of the set $z z^{*}=I$ is

$$
\begin{aligned}
& z_{1}=r e^{i \theta_{1}}, \\
& z_{2}=\left[1-r^{2}\right]^{1 / 2} e^{i \theta_{2}}, \\
& z_{3}=\left[1-r^{2}\right]^{1 / 2} e^{i \theta_{3}}, \\
& z_{4}=-r e^{i\left(-\theta_{1}+\theta_{2}+\theta_{3}\right)},
\end{aligned}
$$

$\left(0 \leqq r \leqq 1,0 \leqq \theta_{j} \leqq 2 \pi, j=1,2,3\right)$.

Proof. If we set $z_{j}=r_{j} e^{i \theta_{j}}$, then $z z^{*}=I$ if and only if $r_{1}^{2}+r_{2}^{2}=1$, $r_{3}^{2}+r_{4}^{2}=1, r_{1} r_{3}+r_{2} r_{4} \cos \alpha=0, r_{2} r_{4} \sin \alpha=0\left(\alpha=-\theta_{1}+\theta_{2}+\theta_{3}-\theta_{4}\right)$. The last equation implies $r_{2}=0, r_{4}=0$ or $\alpha=n \pi(n=0, \pm 1, \cdots)$. Now $r_{2}=0$ implies $r_{1}=1, r_{3}=0, r_{4}=1$. However the set

Presented to the Society, April 20, 1956 under the title Orthogonal systems in matric spaces. Preliminary report; received by the editors August 2, 1958 and, in revised form, September 29, 1958.

1 The research in this paper was done while the author was the holder of an A.A.U.W. National Fellowship in 1954-1955. 


$$
E\left[z \mid z_{1} \bar{z}_{1}=1, z_{4} \bar{z}_{4}=1, z_{2}=z_{3}=0\right]
$$

is the Cartesian product of 2 circles lying in the planes $\operatorname{Re} z_{2}=\operatorname{Re} z_{3}$ $=\operatorname{Im} z_{2}=\operatorname{Im} z_{3}=0$ and hence is a two-dimensional subset of the fourdimensional set $B$ and as such may be disregarded in the integration over $B$. Similarly if $r_{4}=0$. Thus we may take $\alpha= \pm \pi$. Hence $r_{1} r_{3}$ $=r_{2} r_{4}$, also $r_{2}=r_{3}$ and $r_{1}=r_{4}$. Setting $r_{1}=r, \theta_{4}= \pm \pi-\theta_{1}+\theta_{2}+\theta_{3}$, (1) follows.

Now

$$
d V=i d z_{1} d z_{2} d z_{3} d z_{4} / \operatorname{det}^{2} z=i J d r d \theta_{1} d \theta_{2} d \theta_{3} / \operatorname{det}^{2} z
$$

[7], $J$ being the Jacobian of $z$ with respect to $r$ and $\theta$. But

$$
J=-2 i r e^{2 i\left(\theta_{2}+\theta_{3}\right)},
$$

and

$$
\operatorname{det} z=-e^{i\left(\theta_{2}+\theta_{3}\right)}
$$

so that

$$
(f, g)=2 \int_{0}^{1} \int_{0}^{2 \pi} \int_{0}^{2 \pi} \int_{0}^{2 \pi} f \bar{g} r d r d \theta_{1} d \theta_{2} d \theta_{3}
$$

In order to construct the ONS we must evaluate integrals of the type

$$
a_{j k}=\left(P_{j}, P_{k}\right)
$$

where

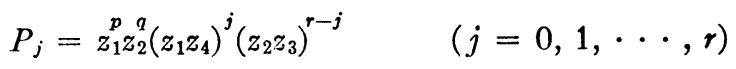

(cf. Lemma 3.2). By (1) and (2)

$$
a_{j k}=(-1)^{j+k} 16 \pi^{3} \int_{0}^{1} r^{1+2 p+2 j+2 k}\left(1-r^{2}\right)^{q+2 r-j-k} d r .
$$

The substitution $t=r^{2}$ transforms $a_{j k}$ into Dirichlet's integral which has the value [8]

$$
a_{j k}=\frac{(-1)^{i+k} 8 \pi^{3}(p+j+k) !(Q-j-k) !}{(p+Q+1) !}
$$

$$
(Q=q+2 r)
$$

3. System of homogeneous polynomials. Let

$$
P(z)=\prod_{j=1}^{4} z_{j}^{p_{j}} \quad Q(z)=\prod_{j=1}^{4} z_{j}^{q_{j}}
$$


$p_{j}$ and $q_{j}$ being non-negative integers. By means of (2.1) and (2.2) it is seen that $(P, Q) \neq 0$ if and only if

$$
\begin{aligned}
& p_{1}-q_{1}=a, \\
& p_{2}-q_{2}=-a, \\
& p_{3}-q_{3}=-a, \\
& p_{4}-q_{4}=a,
\end{aligned}
$$

where $a$ is an integer. ${ }^{2}$ Thus setting $q_{1}=p, p_{2}=q, p_{3}=r, q_{4}=s$, we get

Lemma 1. Let $P$ and $Q$ be powers in $z_{j}$. The inner product $(P, Q) \neq 0$ if and only if

$$
\begin{aligned}
& P(z)=\underset{z_{1} z_{2} z_{3} z_{4}^{8}\left(z_{1} z_{4}\right)^{a},}{p{ }^{q} r}, \\
& Q(z)=z_{1} z_{2} z_{3} z_{4}\left(z_{2} z_{3}\right)^{a},
\end{aligned}
$$

$(p, q, r, s=0,1,2, \cdots ; a \geqq \sup (-p,-q,-r,-s))$.

Lemma 2. Let

$$
\begin{aligned}
& P_{p q r}^{(1 j)}(z)=P_{1 j}(z)=z_{1}^{p q} z_{2}\left(z_{1} z_{4}\right)^{j}\left(z_{2} z_{3}\right)^{r-j} \\
& P_{p q r}^{(2 j)}(z)=P_{2 j}(z)=z_{1}^{p} z_{3}\left(z_{1} z_{4}\right)^{j}\left(z_{2} z_{3}\right)^{r-j}, \\
& P_{p q r}^{(3 j)}(z)=P_{3 j}(z)=z_{4}^{p} z_{2}\left(z_{1} z_{4}\right)^{j}\left(z_{2} z_{3}\right)^{r-j} \\
& P_{p q r}^{(4 j)}(z)=P_{4 j}(z)=z_{4} z_{3}\left(z_{1} z_{4}\right)^{j}\left(z_{2} z_{3}\right)^{r-j}
\end{aligned}
$$

$(j=0,1, \cdots, r ; p, q, r=0,1,2, \cdots)$. Then $\left(P_{p q r}^{(i j)}, P_{\left(p^{\prime} q^{\prime} r^{\prime}\right)}^{\left(t^{\prime} j^{\prime}\right)} \neq \neq 0\right.$, if and only if $i=i^{\prime}, p=p^{\prime}, q=q^{\prime}$ and $r=r^{\prime}$.

Proof. Let $P(z)$ be given by (1). Suppose $p_{4}=\min \left(p_{1}, p_{4}\right)$, $p_{3}=\min \left(p_{2}, p_{3}\right)$. Define $j=p_{4}, p=p_{1}-p_{4}, r=p_{3}+p_{4}, q=p_{2}-p_{3}$. This expresses $P$ in the form (2.3). A power $Q=z_{1}^{q_{1}} z_{2}^{q_{2}} z_{3}^{q_{3}} z_{4}^{q_{4}}$ is nonorthogonal to $P$ if and only if, in case $q_{4} \leqq p_{4}$,

$q_{1}=p+j-a, q_{2}=q+r-(j-a), q_{3}=r-(j-a), q_{4}=j-a$ $(a=0,1, \cdots, j)$, or, in case $q_{4} \geqq p_{4}$, $q_{1}=p+j+a, q_{2}=q+r-j-a, q_{3}=r-j-a, q_{4}=j+a$ $(a=0,1, \cdots, r-j)$, that is, in case

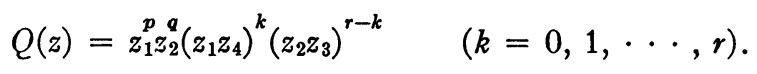

This proves (i) of Lemma 2 and similarly for the other parts.

2 Conditions (2) may be generalized to $n$ by $n$ matrices if integration is over $D$, thus giving a procedure for subdividing the powers of a fixed degree into non-orthogonal subsets. 


\section{ONS of homogeneous polynomials.}

TheOREM. An ONS of homogeneous polynomials on D orthonormalized with respect to the inner product (1.1) is

$$
\phi_{p q r}^{(i j)}(z)=N_{p y r}^{(j)} P_{i j}(z) F(-j,-j-p ; Q+2-2 j ; Z)
$$

$(i=1, \cdots, 4 ; j=0, \cdots, r ; p, q, r=0,1,2, \cdots ; q \neq 0$ for $i=2$, $p \neq 0$ for $i=3, p q \neq 0$ for $i=4)$ where $F$ is the hypergeometric function and $Z=z_{2} z_{3} / z_{1} z_{4}$. The normalizing factor is

$$
N^{(j)}=N_{p q r}^{(j)}=\left[\frac{j+1}{8 \pi^{3}}\left(\begin{array}{c}
p+Q+1-j \\
p+j
\end{array}\right)\left(\begin{array}{c}
Q+1-j \\
j+1
\end{array}\right)\right]^{1 / 2} .
$$

Proof. The hypergeometric function has the finite expansion

$$
\text { (a) } F(-j,-j-p ; Q+2-2 j ; Z)=\sum_{\nu=0}^{j} C_{j, j-\nu} Z^{\nu}
$$

$$
\text { (b) } C_{j \nu}=\frac{\left(\begin{array}{l}
j \\
\nu
\end{array}\right)\left(\begin{array}{c}
p+j \\
j-\nu
\end{array}\right)}{\left(\begin{array}{c}
Q+1-j-\nu \\
j-\nu
\end{array}\right)} \text {. }
$$

Orthogonality. From (3.2) and Lemma $2\left(\phi_{p q r}^{(i j)}, \phi_{s t u}^{(k)}\right)=0$ if $l \neq i$. Similarly $\left(\phi_{p q r}^{(i j)}, \phi_{s t u}^{(i k)}\right)=0$ unless $p=s, q=t$ and $r=u$. It only remains to show that

$$
\left.I_{j k}=\left(\phi^{(i j)}\right), \phi^{(i k)}\right)=0 \quad \text { for } j \neq k,
$$

$\left(\phi^{(i j)}=\phi_{p q r}^{(i j)}\right)$. Without loss of generality we may assume $k<j$ and $i=1$. Then

$$
I_{j k}=N \sum_{\nu=0}^{j} \sum_{\mu=0}^{k} C_{j, j-\nu} C_{k, k-\mu} a_{j-\nu, k-\mu}
$$

$\left(N=N_{p q r}^{(j)} N_{p q r}^{(k)}\right)$, or replacing $\nu$ by $j-\nu$ and $\mu$ by $k-\mu$,

$$
I_{j k}=N \sum_{\mu=0}^{k} C_{k \mu} I_{j \mu}^{\prime}
$$

where

$$
I_{j \mu}^{\prime}=\sum_{\nu=0}^{j} C_{j \nu} a_{\nu \mu}
$$

We prove (4) by induction on $k$ for fixed $j$. Specifically we show that 
$I_{j 0}=N I_{j 0}^{\prime}=0$ and that $I_{j \sigma}^{\prime}=0$ for $\sigma=0,1, \cdots, k-1$ implies $I_{j \mathfrak{k}}^{\prime}=0$. Hence $I_{j k}=0$.

(i) For $k=0$,

$$
\begin{aligned}
I_{j 0} & =N C_{00} \sum_{\nu=0}^{j} C_{j \nu} a_{\nu 0} \\
& =A_{j} S_{j 0},
\end{aligned}
$$

where

$$
A_{j}=(-1)^{i} 8 \pi^{3} N \frac{(p+j) !(Q+1-2 j) !}{(Q+p+1) !}
$$

and

$$
S_{j 0}=\sum_{\nu=0}^{j}(-1)^{j-\nu}\left(\begin{array}{l}
j \\
\nu
\end{array}\right) \frac{(Q-\nu) !}{(Q+1-j-\nu) !} .
$$

By using the binomial theorem and then differentiating the expression

$$
D_{j-1, k}(x, y)=\frac{\partial^{j-1}}{\partial x^{k} \partial y^{j-1-k}} x^{p+k} y^{Q-j-k}(x-y)^{j},
$$

(defined for $k<j$ ), we find that

$$
S_{j 0}=D_{j-1,0}(1,1) \text {. }
$$

Upon differentiating (9) we see that $D_{j-1, k}(x, y)$ equals $x-y$ times a polynomial in $x$ and $y$. Hence $D_{j-1, k}(1,1)=0$ for all $k<j$. Thus $S_{j 0}$ and $I_{j 0}$ are both 0 .

(ii) Let $k$ be such that $0 \leqq k \leqq j-1$. Assuming by induction hypothesis that $I_{j_{\sigma}}^{\prime}=0$ for $\sigma=0,1, \cdots, k-1$, we find by using the binomial theorem on $D_{j-1, k}(x, y)$ and then differentiating that $I_{j k}$ is a multiple of $D_{j-1, k}(1,1)$. Thus $I_{j \mathbf{k}}^{\prime}$ and $I_{j k}$ are 0 and the set (1) is orthogonal.

Normality. We have to show that $I_{j j}=1$. By (5) and the fact that $I_{j \mu}^{\prime}=0$ for $\mu=0,1, \cdots, j-1$,

$$
I_{j j}=N C_{j j} I_{j j}^{\prime}=N \sum_{v=0}^{\prime} C_{j \nu} a_{v j},
$$

which by (2.4), (3b) and (7) has the value

$$
A_{j} \sum_{\nu=0}^{j}(-1)^{\nu}\left(\begin{array}{l}
j \\
\nu
\end{array}\right) \frac{(p+\nu+1) \cdots(p+\nu+j)}{Q+1-j-\nu} .
$$


Now the expression

$$
T_{j}=(-1)^{i} A_{j} \frac{\partial^{j}}{\partial x^{j}}\left\{x^{p+j} \int_{0}^{1} y^{Q-2 j}(x-y)^{i} d y\right\}
$$

can be shown to equal $I_{j j}$ when $x=1$ by first using the binomial theorem and then performing the integration and differentiation. On the other hand integrating $T_{j}$ iteratively, then differentiating and setting $x=1$, we find that only one term is different from 0 and its value is

$$
(-1)^{j} A_{j} \frac{j !(Q+p+1) !(Q-2 j) !}{(Q+p+1-j) !(Q+1-j) !}
$$

which equals 1 . Thus (1) is an ONS.

By analogous methods but much more complicated computations it may be shown that the system

$$
\psi_{p q r}^{(i j)}=M_{p q r}^{(j)} P_{i j}(z) F(-j,-j-p ; Q+4-2 j ; Z)
$$

$(i=1, \cdots, 4 ; j=0, \cdots, r ; p, q, r=0,1,2, \cdots ; q \neq 0$ for $i=2$, $p \neq 0$ for $i=3, p q \neq 0$ for $i=4)$ where

$$
\begin{aligned}
M_{p q r}^{(j)}= & {\left[\frac{4(j+1)}{\pi^{4}}\left(\begin{array}{c}
r-j+2 \\
2
\end{array}\right)\left(\begin{array}{c}
q+r-j+2 \\
2
\end{array}\right)\right.} \\
& \left.\left(\begin{array}{c}
Q+p+3-j \\
p+j
\end{array}\right)\left(\begin{array}{c}
Q+3-j \\
j+1
\end{array}\right)\right]^{1 / 2}
\end{aligned}
$$

forms an ONS on $D$ with respect to the inner product

$$
\int_{D} f \bar{g} d V_{D}
$$

(Cf. [5], where some of the calculations are carried out and the general procedure is explained.)

5. Completeness. Hua [4] has proved that the set of powers (3.1) is complete on $D$ for functions of class $L^{2,3}$ that is, if $f$ is an analytic function with finite norm $\left[\int_{D}|f|^{2} d V_{D}\right]^{1 / 2}$, on $D$, and

$$
\int_{D} f \bar{P} d V_{D}=0, \quad\left(p_{j}=0,1,2, \cdots ; j=1, \cdots, 4\right),
$$

then $f=0$ on $D$. Now each orthogonal set of $(4.1)(j=0, \cdots, r)$ is

\footnotetext{
${ }^{3}$ Class $L^{2}$ was first introduced by Bergman (cf. [1]).
} 
formed from $r+1$ distinct powers of the type described in Lemma 2 . Thus

$$
\phi_{i j}=\sum_{\nu=0}^{j} C_{j p} P_{i v} \quad\left(j=0, \cdots, r ; C_{j j} \neq 0\right) .
$$

Consequently

$$
P_{i j}=\sum_{\nu=0}^{j} d_{j \nu} \phi_{i \nu}
$$

Furthermore the proofs of Lemmas 1 and 2 show that every power $P$ belongs to one and only one set $\left\{P_{i j}\right\}(i=1, \cdots, 4)$. Consequently $\{\phi\}$ is complete with respect to functions of class $L^{2}$ on $D$. Similarly for the ONS (4.10).

6. Expansion theorems. By a result due to Bergman [1] any function $f$ of class $L^{2}$ on $D$ has an orthogonal development

$$
S=\sum_{n=0}^{\infty} a_{n} \psi_{n}, \quad a_{n}=\int_{D} f \psi_{n} d V
$$

and (1) converges absolutely and continuously to $f$ in $D\left(\left\{\psi_{n}\right\}\right.$ is any convenient ordering of the ONS (4.10)). Also if $\left\{a_{n}\right\}$ is an arbitrary sequence of constants such that $\sum\left|a_{n}\right|^{2}$ converges, then the series $S$ converges absolutely and continuously in $D$. In our case this gives a theorem on the convergence of a series of hypergeometric functions.

Also (4.10) gives an expansion for the Bergman kernel function [6]

$$
K(z, t)=12 /\left[\pi^{4} \operatorname{det}^{4}\left(I-z t^{*}\right)\right],
$$

namely,

$$
K(z, t)=\sum_{n=0}^{\infty} \psi_{n}(z) \psi_{n}\left(t^{*}\right),
$$

in terms of hypergeometric functions for all $z, t$ in $D$. Analogous results hold for the Szegö kernel,

$$
1 /\left[8 \pi^{3} \operatorname{det}^{2}\left(I-z t^{*}\right)\right]
$$

[2], related to the ONS (4.1).

\section{REFERENCES}

1. S. Bergman, The kernel function and conformal mapping, Mathematical Surveys, vol. 5, American Mathematical Society, 1950.

2. S. Bochner, Group invariance of Cauchy's formula in several variables, Ann. of Math. vol. 45 (1944) pp. 686-707. 
3. L. K. Hua, On the theory of functions of several complex variables. I. On a complete orthonormal system in the hyperbolic space of rectangular matrices, Dokl. Akad. Nauk SSSR (N.S.) vol. 93 (1953) pp. 775-777 [Math. Rev. 15 (1954) p. 617] and J. Chinese Math. Soc. vol. 2 (1953) pp. 288-323 [Math. Rev. 17, 1956, p. 191].

4. - Harmonic analysis of the classical domain in the study of analytic functions of several complex variables, Mimeographed Lecture Notes, about 1956.

5. J. Mitchell, An example of a complete orthonormal system and the kernel function in the geometry of matrices, Proceedings of Second Canadian Mathematical Congress, Vancouver, 1949, pp. 155-163.

6. - The kernel function in the geometry of matrices, Duke Math. J. vol. 19 (1952) pp. 575-584.

7. - Potential theory in the geometry of matrices, Trans. Amer. Math. Soc. vol. 79 (1955) pp. 401-422.

8. E. T. Whittaker and G. N. Watson, $A$ course of modern analysis, Cambridge, Cambridge University Press, 1948.

Pennsylvania State University 